In addition to the more formal business before the commission Dr Baum has undertaken to arrange a symposium on image tubes. This should be a most valuable part of the proceedings at Dublin.

REFERENCES

(I) Trans. I.A.U. 8, 360-I, I954.

(2) Trans. I.A.U. 8, $381,1954$.

(3) H. L. Johnson, $A p . J .116,272,1952$.

(4) O. J. Eggen, L.O.B. no. 532.

(5) H. L. Johnson and W. W. Morgan, Ap. J. 117, 313, 1953.

(6) Trans. I.A.U. 8, 378, 1954.

(7) Cape Mimeograms, nos. 2 and 3, 1953.

(8) A. Beer, R. O. Redman and G. G. Yates, Memoirs Royal Astronomical Society, 67, pt. I, I954.

(9) E. Rybka, Acta Astronomica, ser. C, 5, 40-3.

(10) Bergedorfer Spectral Durchmusterung, 5 .

(I I) Th. Walraven and A. D. Fokker, B.A.N. no. 435, 1952.

(12) V. P. Tsesevich, Publ. Central Astr. Obs. Acad. Sci. Ukr. S.S.R. 1, 3, 1953.

(13) V. B. Nikonov, Bull. Abastumani Obs. no. r4, 1953.

(14) O. Eklöf, Ark. Astr. x, no. 27 (Uppsala Medd. no. 106), 1953.

(15) J. Stebbins and A. E. Whitford, $A p . J$. 102, 318, 1945.

(16) G. E. Kron, H. S. White and S. C. B. Gascoigne, $A p . J$. 118, 502, 1953.

(I7) G. E. Kron and J. L. Smith, $A p . J$.113, 324, 1951.

(18) H. E. Butler, The Observatory, 73, 80, 1953.

APPENDIX

\title{
REPORT ON STUDIES OF SCINTILLATION
}

\section{Definition}

\section{Introduction}

It is assumed that the word 'scintillation' includes all effects connected with atmospheric seeing, whether the object viewed be a star or an extended body.

Scope of report

This review covers roughly the last ten years and is not intended to be comprehensive.

\section{General}

Astronomers have been forced to take notice of scintillation because of its nuisance value. A considerable amount of observational and theoretical work has already been carried out. Nevertheless, the main interest that astronomers have in fully understanding scintillation is that they may thereby be in a better position to try and overcome its ill effects. On the other hand, the study of scintillation is of great interest to meteorologists. Since observation usually requires the telescopic equipment of an astronomical observatory, it would appear that the active observers of scintillation will remain astronomers, although much of the interpretation of the results will of necessity be done by meteorologists.

\section{Observations}

Between I948 and I954 several series of observations were made of the intensity variations of the telescopic image of a star, particularly by Boutet (x), Butler (2), Ellison and Seddon(3), Goldstein (4), Hosfeld (5), Mikesell, Hoag and Hall (6), Nettleblad(7) and 
Siedentopf and Elsässer (8). In general the methods were the same. A photomultiplier cell was placed near the focus of the telescope and the light from the chosen star was directed in some manner on to the photo-cathode of the cell, the output being recorded either directly or, in the case of Mikesell, Hoag and Hall, being fed into a wave analyser.

In the publications by Butler and by Ellison and Seddon, variations of the recorded intensity fluctuations with altitude of star, and also with aperture of telescope, were demonstrated. Both papers mention rapid intensity fluctuations, Butler reporting up to about 500 peaks per second. Ellison and Seddon also gave traces for light from planets, and made derivations of the height of the region of the atmosphere causing the scintillation. Nettleblad gives the results of extended observations correlating scintillation with stellar altitude and aperture and also gives a review of other work. This paper contains what is probably the most complete bibliography of the subject. The photo-electric work of Mikesell, Hoag and Hall covers the above points more completely still and extends the work to cover polarization and other variable parameters. The use of their wave analyser allowed a ready presentation of their results. They also made simultaneous recordings in different colours.

Hosfeld (s) shows that brightness scintillation and image motion are essentially independent phenomena. Siedentopf and Elsässer (8) made a series of observations at the Jungfraujoch observatory $3600 \mathrm{~m}$. above sea-level. They examined the effects of different zenith distances, apertures and instrumental time constants and showed that the amplitude of scintillation was distinctly smaller than for similar observations at sea level.

Photographic observations on atmospheric seeing have been made by Gaviola (9), Hansson, Kristenson, Nettleblad and Reiz (ro), Zwicky(ri) and also by Mikesell, Hoag and Hall (6).

Gaviola photographed wave patterns on the mirror of a 60 in. telescope and also studied the distribution of light in photographic star trails. Hansson et al. were concerned in measuring the steadiness of seeing by numerous photographs of images formed from two parallel beams. They obtained correlations with stellar elevation and compared results for different stations.

Mikesell, Hoag and Hall have taken ciné photographs of the wave patterns on the mirror of a 40-inch telescope and have demonstrated how the patterns are correlated with winds at different heights. Further ciné photographs of 'Hartmann' images show the lateral movements of the images formed by beams falling on different parts of the mirror.

Zwicky obtained trails of stellar spectra and showed some of the characteristics of colour scintillation. He observed a maximum of rooo peaks of intensity per second. He has given preliminary data (( 2 ) and private communications) on extended observations using equipment which has a time resolution of up to $0.5 \times 10^{-6} \mathrm{sec}$. His current programme includes a determined attack upon the distribution of the atmospheric irregularities with height.

\section{Theoretical work}

Papers concerned with the theoretical aspect of scintillation-the distribution and position of inhomogeneities in the atmosphere and their effect on a traversing light beam-have been published by Chandrasekhar(13), Keller(14), Keller and Hardie (xs), Little (16), Megaw (r7) and van Isacker(18). In addition, in some of the papers already mentioned, attempts are made to interpret the observed results. Apart from Megaw, who regards scintillation as being due to changes in refractive index throughout the atmosphere, all interpret the scintillation as arising in a relatively thin layer of disturbed air. A joint meeting on 'Scintillation' was held in London by the Royal Astronomical Society and the Royal Meteorological Society in 1953 and the report of that meeting (I9) represents one attempt to promote the joint interests of meteorologists and astronomers. 


\section{Conclusions}

Observations and discussions to hand, while greatly advancing our knowledge of atmospheric irregularities and astronomical seeing, leave considerable work still to be done. The presence of rapid intensity variations in a stellar image, although unexpected, will probably not affect any branches of photographic photometry, as far as present-day accuracy is concerned. Due allowance, however, must be made for it in photo-electric work. In any form of automatic telescope guiding, very considerable ingenuity must be exercised in order to overcome the effects of scintillation. We are not at present able to decide whether rapid guiding of a photographic plate in either two or three dimensions would improve the images on that plate.

As far as stellar spectroscopy is concerned, scintillation enlarges the stellar image until its diameter is, in the majority of instruments, much greater than the slit width that gives optimum resolution. Consequently we have either to accept the loss of light at the slit or widen the slit and accept the loss of resolving power. It would appear that further attempts should be made to increase instrumental efficiency, particularly by designing instruments to work with the image diameters that are actually found in practice.

Our knowledge concerning the distance from the observer of the effective atmospheric irregularities is very incomplete. It would appear that the most important matter for astronomers is the determination of the proportion of scintillation which is produced within a few hundred metres of the telescope. If it should be a large percentage of the whole, then it may be possible by siting, engineering, or otherwise, materially to improve the seeing at an observatory.

Some of the effects of disturbances very near to the telescope have been examined by Hosfeld (s).

H. E. Butler

\section{REFERENCES}

(1) R. Boutet, 'Enregistrement photoélectrique de la scintillation des étoiles ', Ann. Geophys. 5, 3 ro, r949.

(2) H. E. Butler, 'Observations of stellar scintillation', Proc. R. Irish A.cad. 54, A, 321, 1952. (Dunsink Obs. Contrib. no. 4.)

(3) M. A. Ellison and H. Seddon, 'Some experiments on the Scintillation of stars and planets', Mon. Not. R. Astr. Soc. I12, 73, 1952. (Edinburgh Commun. no. 4.)

(4) E. Goldstein, 'The measurement of fluctuating radiation components in the sky and atmosphere'. Part I, Naval Research Lab., Report N-3462 (1949); Part 2, N.R.L. Report 37 Io (1950). (Abstract in J. Opt. Soc. Amer. 40, 795, 1950.)

(5) R. Hosfeld, 'Comparisons of stellar scintillation with image motion', J. Opt. Soc. Amer. 44, 284, 1954. (Perkins Obs. Contrib. ser. II, no. 3.)

(6) A. H. Mikesell, A. A. Hoag and J. S. Hall, 'The scintillation of starlight', J. Opt. Soc. Amer. 41, 689, I95I.

(7) F. Nettelblad, 'Studies of astronomical scintillation', Lund Medd. 2nd ser., no. I30, I953.

(8) H. Siedentopf and H. Elsässer, 'Szintillationsuntersuchungen am Jungfraujoch', $Z$. $A p$. 35, 21, I954. (Tubingen Mitteilungen, nó. I4.)

(9) E. Gaviola, 'On seeing, fine structure of stellar images, and inversion layer spectra', Astr. J. 54, 155, 1949. (Cordoba, Tirada Aparte, no. 34.)

(ro) N. Hansson, H. Kristenson, F. Nettelblad and A. Reiz, 'On the atmospheric unsteadiness', Ann. d'Astrophys. 13, 275, 1950. (Lund Medd. ser. II, no. I29.)

(I I) F. Zwicky, 'Seeing', Publ. Astr. Soc. Pacif. 62, I50, I950. (Mt Wilson and Palomar Observatories Reprint, no. 32.)

(12) F. Zwicky, 'Scintillations of stars and indirect meteorology', J. Amer. Rocket Soc. Nov.-Dec. I953. 
(13) S. Chandrasekhar, 'A statistical basis for the theory of stellar scintillation', Mon. Not. R. Astr. Soc. 112, 475, 1952.

(I4) G. Keller, 'Astronomical seeing and its relation to atmospheric turbulence', Astr. J. 58, II3, I953. (Contrib. Perkins Obs. ser. II, no. I.)

(15) G. Keller and R. H. Hardie, 'Experimental verification of a recently proposed theory of astronomical seeing', Astr. J. 59, 105, 1954. (Contrib. Perkins Obs. ser. II, no. 2.)

(16) C. G. Little, "A diffraction theory of the scintillation of stars on optical and radio wavelengths', Mon. Not. R. Astr. Soc. III, 289, I95I. (Manchester, Jodrell Bank Reprint, no. 5 I.)

(I7) E. C. S. Megaw, 'Scattering of electromagnetic waves by atmospheric turbulence: Stellar scintillation and the spectrum of turbulence in the free atmosphere', Nature, I66, I I00, I950.

(I8) J. van Isacker, 'La Scintillation des Etoiles', Publ. Inst. R. Meteorol. Belg. B, no. 8, I953.

(19) 'Report of, joint meeting of the R.A.S. and R.M.S.', Quart. J. R. Met. Soc. 80, 24I-60, I954.

\section{Report of meetings}

President: Prof. W. M. H. Greaves.

SeCretary: Dr H. E. Butler.

The Commission met on 30 August at 9 a.m.

The Draft Report was adopted as a basis for discussion.

A letter was read from Wesselink urging the desirability of preparing special charts for the Selected Areas. Stoy and Baum said that some charts of this kind would be of value. The President remarked that before financial proposals were put forward the Commission would have to have before it a definite scheme. It was agreed that Stoy, Baum and Wesselink should discuss the matter before the tenth General Assembly and that if possible they should communicate definite proposals to the General Secretary before then.

On the question of whether the Commission should continue to give hospitality to the subject of 'Scintillation', Butler advocated the formation of a new Commission. Minnaert agreed that the subject needed organized co-ordination and suggested either a new commission or, at least, a new sub-commission. Oosterhoff referred to the impending reorganization of Union membership and thought that the Executive would be, for the time being, opposed to the creation of new commissions. The discussion was continued by Melnikov, Redman, J. S. Hall and Bok. It was felt that there should be at least a new sub-commission and that 'Scintillation' was too narrow a title. It was resolved that the Executive should be asked to create a sub-commission on 'Seeing' to Commission 25.

In inviting discussion on the first section ('Standard Systems') of the Report the President stated that it aimed at setting forth the background against which the terms of reference of the sub-commissions were drawn up. In the absence of Eggen he showed a diagram from Eggen's paper mentioned in the Report. This diagram exhibited a comparison of Eggen's $P-V$ and Johnson and Morgan's $B-V$ (see Report, p. 339). For the later type stars the relation between these two colour systems was not one to one but depended on luminosity. The blue filters used in these two-colour systems were both ultra-violet-free but differed as regards the cut off on the long-wave-length side. Baum said that Eggen's work had amplified the work of Johnson and Morgan.

Weaver pointed out that the conversion of magnitudes and colours was an important aspect of photometry. The value of an observer's work was not a maximum if it could not accurately be compared with that of others, particularly in the case of accurate photo-electric magnitudes and colours. Accurate conversion was not possible if the two systems being compared were based on different wave-length ranges. Particular 
amculty was encountered it the uutra-violet on the snort-wave side of $3800 \mathrm{~A}$ or $3900 \mathrm{~A}$ was included in the blue magnitudes. For ordinary magnitudes needed for statistical purposes (and he made it ciear that he was at no time referring to magnitudes for astrophysical use) it was of considerable advantage if all observers used approximately the same wave-length ranges for their work and referred it to a standard sequence of stars, thus making possible accurate conversions between the work of different observers. Suitable magnitude sequences must be available for such comparisons. The use of proper filters for approximate limitations of wave-length bands to specified ranges would appear highly desirable in the determination of ordinary magnitudes.

In answer to a question, W. Becker stated that his proposal for the construction of standard filters had fallen through because makers could not guarantee permanence of standardization.

The Commission then considered the report of the sub-commissions.

Bok considered that although Becker's three-colour system would remain useful for faint photometric work, the system must be considered as secondary to the Johnson and Morgan $U, B, V$ system and the I953 $S$ system which the Cape observers had tied up so well. He also wanted the Commission to make suggestions regarding the most suitable filter combinations to reproduce $U, B$ and $V$. Weaver supported Bok and also would have liked the sub-commission to recommend the general use of a single set of standardsthe $U, B, V$ system. Becker spoke of his own system of colours. He pointed out that he found that the $p g$ region extended too far into the ultra-violet and so included luminosity effects for late-type stars. Therefore he preferred to cut off the photographic region so that the equivalent wave-length would be about $4700 \AA$. The three regions he proposed would then be conveniently covered by existing colour filters and plates.

The President then asked for views on the N.P.S. system. Redman said that many good and bad observations based on this system were in existence and that they should not lightly be thrown away. He would prefer that photo-electric photometry should be done with a monochromator and not with filters.

Fellgett disagreed that failure of systems to be convertible was a 'trouble' or 'disadvantage'; on the contrary, it was this very fact that enabled photometry to yield information on astrophysical parameters.

Baum said that the $U, B, V$ system was in use at Mt Wilson and Mt Palomar for faint as well as for bright stars, although the $U$ value was not always given. But he agreed that more colours were needed. With the exception of the ultra-violet, he thought that conversion would be straightforward. Miss Underhill stressed that the standard systems to be recommended depended upon the use to be made of the material: for statistical studies certain wide bands may be useful, while for astrophysical studies multi-colour systems and the differences between these systems were all of interest and all gave different information.

Stoy said that $(U-B)$ colours were now being determined for the stars in the $E$ region sequences and that it was intended that ultimately the $S$ system should be a full three(or more) colour system. The $S$ system was not to be regarded as a permanent alternative to the ather systems; it could be converted to form the southern extension of whichever of the northern systems was ultimately adopted as standard. No immediate change was contemplated in $S P v$, but it was proposed to revise $S P g$ by applying corrections to eliminate the small ultra-violet 'leak'. Only a few individual stars with spectral types between Ao and $\mathrm{Ko}$ would be altered by more than $\mathrm{o}^{\mathrm{m} \cdot 005}$, but for Bo stars the correction would amount to $\mathrm{o}^{\mathrm{m} \cdot 05}$. The $(U-B)$ colours measured with the Cape refractors could be closely correlated with the $(U-B)$ colours of the Johnson-Morgan system by a relationship of the form

$$
(U-B)_{J}=2.94(U-B)_{C}-0.54(B-Y)_{C}+\text { const. }
$$

Baum pointed out that many 'photographic' observations used a Schott GG-I3 filter (with ultra-violet cut-off) and hence were comparable with the $B$ system. Weaver questioned whether the commission should concern itself with standards for multi- 
colour observations such as those mentioned by Miss Underhill. He did consider, however, that they should be concerned with the standardization of standard magnitudes for statistical work.

W. Becker agreed with Fellgett and Miss Underhill with respect to the importance of disagreements between systems. That was why they had changed from a two-colour to a three-colour system (see Göttingen Acad. of Sci. I946). Fellgett agreed with Weaver on the need to distinguish always between the requirements of statistical and of astrophysical photometry and referred to the advantages of Kron's previous suggestion of measuring magnitudes in a red region centred on the minimum of atmospheric extinction.

Mrs Payne-Gaposchkin considered that photographic work would inevitably continue and stressed the need for data on plate-filter combinations to simulate the $U, B, V$ system. Since instruments would inevitably have slight differences, everyone should check his magnitude system by reference to standard stars which should be well distributed over the sky. Bidelman strongly endorsed this.

Baum remarked that while it may be impracticable to duplicate a given magnitude system exactly, it was always possible to approximate to it sufficiently closely for the resulting transformations to be very simple.

The Commission then adopted the following resolutions:

I. That for standardizing two-colour photometry, observers should use:

(a) A modification of the International $p g, p v$ system,

or (b) the $B, V$ system of Johnson and Morgan,

or $\quad(c)$ in the Southern Hemisphere, the $1953 S$ system.

2. That for standardizing three-colour photometry, observers should for the present use the $U, B, V$ system of Johnson and Morgan.

3. That observers be urged to use a filter (such as the Schott GG-13) to cut out radiation with wave-lengths shorter than $3800 \AA$ whenever 'blue' or 'photographic' magnitudes were being observed.

It was decided to take no action with regard to recommendation 4 of the sub-commission, it being considered that formal endorsement was unnecessary.

It was also resolved to recommend to the Executive that the existing two subcommissions be combined under the title 'Sub-commission on Standard Magnitudes'.

The Commission then adjourned.

The Commission resumed on 3 September at 9 a.m.

The President remarked that the section of the Report setting forth Progress Reports had been necessarily condensed. He hoped that members would expand it in the course of their remarks.

Baum spoke of the work on photon counting now being done with the 200-inch telescope at Mt Palomar. The method was used for the very faint objects. In measuring a blue magnitude $(B)$ of 23.5 or a visual magnitude $(V)$ of 23.0 , approximately one photon per second was counted. At the same time a diaphragm 4 seconds of arc in diameter gave about 40 counts per second from the sky.

In order to obtain a certain prescribed accuracy, it was necessary to count for a time

$$
T=\frac{2 \text { (sky count } / \text { sec. })+(\text { star count } / \text { sec. })}{(\text { probable error in magnitudes })^{2}(\text { star count } / \mathrm{sec} .)^{2}} \text { seconds. }
$$

In half a night they could measure a $24^{\mathrm{m}}$ star with an accuracy better than $0^{\mathrm{m}} \cdot 2$. It was better to measure a number of stars with low accuracy than a few stars more precisely since they could never tell whether the 'sky' included any $25^{\mathrm{m}}$ stars. The method was slightly more sensitive than photography.

Fellgett said that as Redman was not present, he felt he ought to mention the work going on at Cambridge, England. He believed that the first specific suggestion for using pulse counting in astronomy was due to Kron $(A p . J .103,326,1946)$ and it was first demonstrated by Blitzstein. At Cambridge they were interested in pulse counting, not 
so much for long integration times (which could be achieved in other ways) as for improved statistics arising from the fact that each effective photon was given equal weight, whereas in the direct current method this was not so. Yates had published (Mon. Not. R. Ast. Soc. I08, 476, I948) a reasonably thorough evaluation of pulse counters at the telescope. He had measured 123 nearby stars in this way (Mon. Not. R. Ast. Soc. 114, 218, 1954) and the photo-electric part of the $+15^{\circ}$ selected area photometry of Beer, Redman and Yates (Mem. R. Ast. Soc. 67, pt. I, I954) was also done by pulse counting.

Hiltner felt that d.c. methods including integration might be better than pulse counting. In reply Fellgett said that with normal gain-per-stage the unequal amplification of pulses arising from those photons which were effective would be sufficient to reduce the signal/noise power ratio of a d.c. measurement by a factor of about $\sqrt{ } 2$ if the statistics of multiplication were Poissonian (Woodward, Proc. Camb. Phil. Soc. 44, 404, I947). In practice (Lenouvel, Pub. Obs. Haute-Provence, 2, no. 4I, I952), reduction by a further factor of about $\sqrt{ } 2$ occurred, so that a given measurement took twice as long as is in principle necessary.

Baum pointed out that there were methods which brought up the accuracy of pulse counting as compared with the d.c. method. At Mt Palomar they used a 6685 E.M.I. cell with a cathode $\mathbf{I ~} \mathrm{cm}$. in diameter. In reply to a question by Butler, he said that even in summer they had not found it necessary to refrigerate the cell. They were lucky in having an excellent cell.

The President invited Nikonov to say something about photometry in the U.S.S.R. Nikonov said that there were four observatories working on the subject, all using d.c. amplification with Russian-made photomultipliers. One programme which had just been completed showed good agreement with the results of Johnson and Morgan. They were at present developing a method of integrating counts in order to remove seeing troubles. Abastumani Observatory had just completed the observation of I084 stars of types B8-Bg in two colours and with an accuracy of $\mathrm{o}^{\mathrm{m}} \cdot 005$. Their work also included variable stars; and they had discovered independently that the colour of $\beta$ Cep. was variable. Weaver asked whether they had done any three-colour work. Nikonov replied that they had done so for the near stars. They were also working at the moment with a spectrometer, using Hiltner's method.

On being asked to speak, Morton of the Radio Corporation of America described the spectral characteristics of the so-called tri-alkali cathode photomultiplier. This cell very conveniently covered the region of low sensitivity which existed between blue sensitive and infra-red sensitive cells. In fact its sensitivity was as much as roo times that of present ones in the red giving 250 to 300 amps/lumen. The surfaces already existed in the laboratory. Their production depended on economic factors. Fellgett asked whether it was true that the tri-alkali surface had a quantum efficiency of $20 \%$ for semi-transparent cathodes. Morton agreed and said that the antimony-caesium oxide surface could also have a comparable efficiency. In reply to Behr, Morton said that the dark current was ten times that of the $\mathrm{Sb}-\mathrm{CsO}$ cells. J. S. Hall asked what were the prospects of an infra-red photo-multiplier in the near future. Morton replied that there already existed one by Capehart Farnsworth.

Fellgett reported a development by Kron (see Kron and Fellgett, P.A.S.P., 67, 334) intended to exploit the statistical advantage of the simple diode photo-cell. The Kron type of electrometer amplifier is modified by removing the high load resistor in order to eliminate completely its Johnson current-noise. The charge on the grid of the electrometer builds up during the exposure and it is then discharged by an earthing switch. The resultant saw-tooth wave is fed to the recorder through a differentiating circuit so that the presentation is as usual.

A further development is to allow the photo-current to accumulate charge on the isolated anode of the photo-cell, and to connect the electrometer only at the end of the exposure; this avoids mixing grid current with the photo-current until the last moment. With optimum filtering it appears that the r.m.s. error in charge measurement can be made as low as \pm 70 electrons. Whitford said that Kron was still working on this. The 
switching contact was extremely delicate; with the cooled electrometer the grid current was so low that the grid could be left connected during the exposure.

The President then closed this part of the discussion and passed on to the topics for discussion listed on p. 345 of this volume.

\section{Item I. The Zero-point Problem in Stellar Photometry}

Rybka said that photo-electric methods now made it possible to transfer photometric zero points over the whole sky with an accuracy of the order of $\mathrm{o}^{\mathrm{m}} \cdot \mathrm{oz}$. He thought that practical advantage should be taken of this since existing zero points were subject to much larger errors. For an accurate transfer from one part of the sky to another it was necessary to determine the instantaneous atmospheric extinction. At the Rome meeting of the Commission in 1952 he had advocated the establishment of a photometric net of standard stars distributed over the whole sky. This task had, in fact, been undertaken at the Wroctaw Observatory and photo-electric observations of suitable standard stars had been secured since I95I. The scheme involved the selection of two stars near each Kapteyn Selected Area, one of the pair being of spectral type A (B 5 to $\mathrm{A}_{5}$ ) and the other of type $K\left(G_{5}\right.$ to $\left.K_{5}\right)$. The aggregate of these pairs constituted the net and the aim was to determine $I P g$ and $I P v$ magnitudes for each star in the net. The selected standard stars were between 5.5 and 6.5 visual magnitude. The Wroctaw programme would cover the parallels of declination $+75^{\circ},+60^{\circ},+45^{\circ},+30^{\circ}$ and $+15^{\circ}$. Once this standard net had been established it would facilitate the determination of local instantaneous atmospheric extinction; this could then be done by nearly simultaneous observations of standard stars observed at different altitudes. From the determined extinction factors for types $\mathrm{A}$ and $\mathrm{K}$ the factors for other types could be interpolated and the net would serve for the accurate transfer of zero point.

Rybka concluded his remarks by referring to studies of variable stars and he said that for such studies a homogeneous zero point was important. There existed considerable differences in the extreme magnitudes given by various observers for the same variable star. A knowledge of the photometric zero point was not, of course, required for the determination of the shape of a light curve, but it did become important for statistical studies of variables since erroneous zero points could lead to erroneous conclusions. It would be particularly desirable to undertake zero-point revision for the magnitudes of Cepheids.

Stoy agreed with Rybka as to the importance of standards all over the sky in order to facilitate transfer. He pointed out that, in a recent paper $(A . J .60,65$, I955), Eggen had collected well-observed magnitudes and colours for bright stars all over the sky and that these were sufficiently accurate to serve as local standards of zero point. As regards the E region sequences, a special zero-point programme carried out in I952 had fixed their zero points relatively to one another to an accuracy of $0^{\mathrm{m}} \cdot 003$ at the seventh magnitude and relatively to certain stars in the northern hemisphere to an accuracy of $0^{\mathrm{m} \cdot 005}$. W. Becker likewise agreed with Rybka. Any observer could now establish a scale, but not every observer could manage zero points. Hence the need for zero points and standards down to ${ }^{1} \mathrm{O}^{\mathrm{m}}$ or $\mathrm{I}^{\mathrm{m}}{ }^{\mathrm{m}}$. Nikonov agreed with Rybka and Becker and said that the U.S.S.R. astronomers would be participating in this work.

\section{ITEM 2. ATMOSPHERIC EXTINCTION}

Nikonov said that the correct allowance for extinction was a fundamental problem in photo-electric photometry of high precision. But in precision work it was important to take account of the fact that the extinction factor was normally not constant throughout a night, but varied with time. He then presented the results of some calculations made on the assumption that the extinction factor increased uniformly with time throughout a night. These calculations showed that if Bouger's method (which assumes 
constancy of extinction factor) were used, the observations east and west of the meridian would give different values for the extra-atmospheric colour of a star. Bouger's method had to be abandoned since it might lead to quite misleading results at places with a normal climate. Constancy of extinction factor could not be assumed and Nikonov referred to a very elegant method proposed by Smart in 1937. Another method had been developed in the U.S.S.R and was in use at the Crimean, Burakan and Abastumani Observatories (see Nikonov, Bull. Abastumani Obs. no. I4, I953).

Nikonov concluded by saying that at the next General Assembly of the Union, the U.S.S.R. astronomers would like Commission 25 to discuss methods of making allowance for atmospheric extinction in photo-electric work and also to make recommendations.

Fracastoro said that in Catania they had made many measures at sea level and at an elevation of $10,000 \mathrm{ft}$. and had got for three colours the same value on extrapolating to air mass zero. He stressed the need to separate out from accidental errors, real changes in extinction due to sky, smoke, etc.

\section{Item 3. The Need for further Photometry down to the $23^{m}$}

Baum explained that his reason for raising this question was to point out that the methods that had been developed for the 200-inch were equally applicable to smaller telescopes; thus with a 74-inch and a dark sky it should be possible to work down to the 22nd magnitude. This was particularly important in the Southern Hemisphere. Stoy explained that in the Southern Hemisphere they had started work on the bright stars and were systematically working to fainter magnitudes. He remarked that it was not possible to work to the extreme limit either at the Cape because of the city lights or at Pretoria because of the variable glow from the nearby furnaces of the Iscor steel works. The Boyden Station enjoyed a very dark sky and he hoped that its new directors would consider using the 6o-inch reflector for photo-electric observations of faint star sequences. He concluded by remarking how much the photo-electric work at the Cape owed to the visits they had enjoyed from various American astronomers during the past five years and said that anyone who could assist with work on the faint stars at the Cape would be very welcome.

\section{Item 4. The Need in the Southern Hemisphere for Primary and SECONDARY SEQUENCES EXTENDING DOWN TO $20^{\mathrm{m}}$}

Butler explained that the previous item covered this matter. As far as he was concerned the need had arisen when using the ADH telescope in Bloemfontein.

\section{Item 5. The Need for extending Sequences of Red Magnitudes to COVER FAINT Stars}

The President explained that this item was suggested by Dessy. Dessy was not present.

Blanco reported that Commission 33 (Stellar Statistics) had stressed the importance of the publication of red sequences in the clearer areas of the Milky Way down to $\mathbf{I}_{4}{ }^{\mathrm{m}}$.

Hiltner referred to the work at the McDonald Observatory with Lead Sulphide Cells. He was using an Eastman cell in the region $\mathrm{I}-2 \mu$.

Weaver stressed that the importance of red magnitudes was growing. Had not the time come when the establishing of such standards should come before the Commission? Could the sub-commission advise on the best wave-length region? The President suggested postponing further discussion until the next item was discussed. 


\section{Item 6. The Need for Monochromatic Magnitudes of Stars in H $\alpha$ Light}

The President stated that, in a letter to him, Shajn had emphasized the desirability of obtaining red magnitudes in standard regions so as to facilitate the measurement of the absolute brightness of $\mathrm{H} \alpha$ in emission nebulae.

Morton asked if astronomers were interested in photo-conductive cells sensitive at ro $\mu$. There was a window in the atmospheric absorption between $8 \frac{1}{2}$ and $13 \frac{1}{2} \mu$. Whitford asked if these cells were more sensitive than thermo-electric elements. If so they would be of use to planetary observers. He doubted their use for stellar observers. Greaves commented that if they were satisfactorily developed, astronomers would certainly find a use for them.

Fellgett said that usually the greater sensitivity of photo-conductive detectors arose simply from the fact that they were insensitive at longer wave-lengths; no detector working over wide bands in the Io $\mu$ region could be more than a few times more sensitive than existing thermal detectors. The fundamental radiation noise severely limited what could be done at ro $\mu$. A photo-conductor would be exceedingly valuable if it led to an image tube and thus removed the need for scanning.

Baum said that for distant extra-galactic nebulae they had turned to the red where they were using a refrigerated Farnsworth tube with a small cathode. They were able to reach within two magnitudes of what they could in the blue and yellow.

It was agreed to direct the attention of the sub-commission to the discussion on items 5 and 6.

\section{Item 7. The Need for New Observatories in Sites where The Climate is EXCEllent}

Stoy said he believed that Irwin wanted observatories in desert climate. Baum said that this had been discussed at a conference in the U.S.A. The idea was to have one in a desert region in America. Stoy said that he believed that Irwin was now interested in the possibility of such an observatory in the Southern Hemisphere as well.

\section{Item 9. The Problem of Star-counts on Plates obtained with Schmidt Cameras}

Butler stressed that in order to extract the maximum from plates taken with a large Schmidt telescope it was frequently desirable to make complete star counts, and for this, automatic methods were highly desirable. In view of the considerable complications to be overcome in making an electronic instrument, he had experimented with an indirect method which, he believed, showed some promise (Observatory, 73, 80, r953). He asked if any other indirect methods had been explored and for views on the virtues of such an approach. Weaver had doubts about the method giving sufficient accuracy and Keller said that he had found to his cost that the solution of an integral equation such as the method required could present considerable difficulties.

The President next stated that a memorandum by Fellgett had recently been circulated by Redman to members of the Commission. There was now an opportunity to discuss it.

Fellgett then gave a summary of the memorandum in the course of which he had advocated inter alia that photometric results should be expressed in terms of a single magnitude and the appropriate number of colour indices. As regards the single magnitude he advocated that it should be defined by the number of photons per square centimetre per second received outside the atmosphere in a specified standard spectral range. He thought that this standard range should be between the centres of two common lines as sugggested by Hertzsprung (Trans. I.A.U. 6, 407, I938). He also thought it should be near the minimum of atmospheric extinction in the red as suggested by Kron (Trans. I.A.U. 8, 368, 1952). The suggested physical standardization of zero point would involve comparisons between stars and a terrestrial standard. Fellgett said that it did not seem 
obvious to him that this was more difficult than the transfer of a zero point between, for example, the Northern and the Southern sky. The accurate correction to outside the atmosphere would involve spectrophotometry and the difficulties would be minimized by working in the red near the minimum of atmospheric extinction.

Ovenden said that the precise definition of a photometric system was of particular importance in the photometry of eclipsing binary systems. Since the distribution of energy in the combined radiation of an unresolved double star was not, in general, the same as that in the spectrum of a single star of the same mean colour index, the transference from one photometric system to another on the basis of colour index was not permissible. The problem was particularly serious for eclipsing binaries, where the relative contributions of the components to the total light varied throughout the eclipses. The comparison of different series of observations of the same binary in different photometric systems was possible only when the photometric systems were accurately defined.

Stoy said that Fellgett's memorandum raised one of the most important outstanding problems in photometry, namely, the problem of the physical interpretation of what is actually being measured. Several astronomers were already working towards the solution of this problem. One of the biggest difficulties involved was the variability of the atmospheric transmission and he wondered how Fellgett was going to eliminate its effect in practice.

Greaves remarked that in colour temperature work it was well known that relative gradients could be found to a much greater accuracy than absolute gradients. Presumably a similar difficulty would arise in Fellgett's proposed physical standardization of zero point. Hiltner commented on the difficulties to be expected with a standard laboratory source.

Fellgett said that he had worked with laboratory photometric standards and fully agreed that the standardization of the zero point would require great care. Nevertheless, it seemed well worth trying it since it was not obvious that the difficulties would be greater than with present definitions.

The meeting then adjourned until 2.30 p.m.

\section{ADDENDUM}

Subsequent to the Dublin meeting the President of Commission 29 has asked that the attention of members of Commission 25 should be drawn to certain matters arising out of the joint meeting of Commissions 27 and 29. These are: $(a)$ the need for multicolour photometry of symbiotic stars: $(b)$ the need for narrow band photometry of variable stars; and $(c)$ the need for photo-electric studies of variations (possible slow and small) in the light of Be stars, Of stars, shell stars, etc.

On resumption the President explained that this afternoon session would be devoted entirely to the subject of Scintillation on which Butler had prepared the Appendix to the Report: Butler explained that he had been severely restricted for space and had consequently deliberately omitted reference to work on radio. This also accounted for the lack of reference to many important papers. He regretted that a few papers, of which he had been unaware, had not received mention.

Fellgett said that in general, scintillation was concerned with the transmission of electro-magnetic radiation through the atmosphere. There was a well-developed body of theory and practice which had mainly had application to radio and which he considered ought to be included in the references. This was especially so since the earlier papers of the series formed the starting-point of several of the investigations already quoted. $\mathrm{He}$ thought the following papers should be mentioned in addition to the references listed in the Appendix.

J. A. Ratcliffe, Nature, 162, 9, r948.

H. G. Booker, J. A. Ratcliffe and D. H. Shinn, Phil. Trans. A, 242, 579, 1950.

B. H. Briggs and G. J. Phillips, Proc. Phys. Soc. B, 63, 907, 1950. 
A. Hewish, Proc. Roy. Soc. A, 209, 8r, 195I.

J. A. Fejer, Proc. Roy. Soc. $A, \mathbf{2 2 0}, 455,1953$.

M. Ryle and A. Hewish, Mon. Not. R. Ast. Scc. 110, 381, 1950.

E. N. Bramley, Proc. Roy. Soc. $A, 225,515,1954$.

B. H. Briggs, Proc. Phys. Soc. B, 64, 225, I95I.

B. H. Briggs, G. J. Phillips and D. H. Shinn, Proc. Phys. Soc. B, 63, 106, 1950.

A. Hewish, Proc. Roy. Soc. A, 214, 494, 1952.

J. S. Hey, S. J. Parsons and J. W. Phillips, Nature, 158, 234, 1946.

This was approved.

The remainder of the session was concerned with statements by those actively working on scintillation and with discussion of those statements.

Danjon described a visual method using interference principles which gives a direct measure of the turbulence at any moment. (See C.R. 183, I032, I926 and also Vistas in Astronomy, r, p. 377.) He explained that these methods were at the moment in use in South Africa in examining possible observatory sites.

Keller reviewed the recent theoretical work on the general problem of scintillationjumping and spreading of the image, the formation of shadow patterns and the variation in total light at the focus. Most work assumed that the variations were caused by the light passing through regions of different density and hence different refractive index, although quantitative proof was still lacking. He believed that in general it was necessary to use wave theory as opposed to ray theory. It appeared that single scattering was insufficient to explain the phenomena and in spite of the complications we must accept multiple scattering.

There was an important background to the subject by Booker, Ratcliffe and Shinn (Phil. Trans. Roy. Soc. A. 242, 579, I950). Van Isacker's treatment (Inst. Roy. Met. de Belgique, $B$, no. 8, I953) was the only comprehensive mathematical theory that assumed multiple scattering.

His own recent work (unpublished) had calculated the spectrum of pattern element sizes for a number of conditions of height, characteristic element size, effective layer thickness and wave-length of light. Preliminary results indicated that a layer of turbulence $\mathrm{I} 60 \mathrm{~m}$. thick, having r.m.s. temperature fluctuations of $\pm 0^{\circ} \mathrm{I}$ over a characteristic length of $6 \mathrm{~cm}$. and located at an elevation of about $10 \mathrm{~km}$. could produce the observed effects. A thicker layer at a smaller height would also serve. He had recently carried out a detailed mathematical analysis of the effect of telescope aperture on the total intensity of the image ( $J . O p t$. Soc. Am. 45, 845, I955).

He suggested that from the point of view of the theorist, the following were some of the most promising directions for future research:

(I) A detailed and quantitative verification of existing theories of image behaviour and shadow pattern behaviour.

(2) A determination of the values of the parameters describing the atmospheric conditions causing seeing problems, and an investigation of how these parameters vary with weather conditions and geographical location.

(3) An investigation of means of minimizing seeing troubles using our knowledge of the causes (Couder, C.R. Acad. Sci. 236, 780, r953; Babcock, P.A.S.P. 65, 229, 1953).

(4) Application of the techniques of pattern analysis to other fields of astronomy, such as the investigation of solar granulation or of turbulent patterns in gaseous nebulae.

In general discussion, Houtgast observed that from the displacement of the image of a star as a whole in a telescope of small aperture, it followed that the scintillation in this case could be considered as the effect of one or two eddies. Keller replied that it was possible to make allowance for this by a long-term average of intensity in the focal plane. But even in this case the image did not show interference rings. Fellgett thought that there was confusion because the term 'multiple scattering' was sometimes used for a layer which was phase-thick and sometimes for one that was distance-thick. It was known that for the layer to be phase-thick was already sufficient to make the determination 
of anything certain about the structure of the seeing layer exceedingly difficult. Perhaps Hewish could comment on this.

Hewish did not wish the meeting to get involved in detail. He regarded a layer as phase-thin when there was less than one radian of retardation. When the layer was phasethick the frequencies in the scintillation were higher than those in the layer and it was very difficult to get information about the latter.

Kaiser then spoke. He said that he wished to remark on some aspects of weak scattering, i.e. scattering in which the phase deviations impressed on the wave were smaller than one radian.

It was often stated that the scale of the diffraction pattern was the same as that of the irregularities producing it. This was true for a two-dimensional diffracting screen and also for a three-dimensional region in which the refractive index fluctuations could be characterized by a Gaussian auto-correlation function. If, however, the microscale and macroscale (as defined in turbulence theory) of the refractive index fluctuations had widely different values, then it seemed that the microscale in the diffraction pattern would in general have an intermediate value.

J. S. Hall next described work being carried out by Mikesell at the U.S. Naval Observatory. He had recently attempted to correlate scintillation with the height of the tropopause and with wind shear, with little success. There did appear to be some correlation with wind speed at different heights. His observations had extended over a period of five years and were made with telescopes of 4 - and 15 -inch apertures.

The chief point was that with a 4-inch aperture and at frequencies of more than Ioo c.p.s., the degree of scintillation was correlated with maximum wind speed without regard to height and also with wind speeds at definite heights from about $6 \mathrm{~km}$. to at least $\mathrm{r} 6 \mathrm{~km}$. Larger telescopic apertures were required to show similar correlations at lower frequencies. A weak correlation of scintillation and seeing (image motion and size) was found to exist.

Observations of scintillation had been made at Flagstaff by the Lowell and Naval Observatories to determine the height of the layer or layers in which scintillation is produced. The scintillation of lights carried aloft by balloons was measured at both low and high frequencies and compared with the scintillation of stars at similar angular altitudes. Preliminary results indicated that a substantial amount of scintillation was produced above $40,000 \mathrm{ft}$.

In reply to a question by Fracastoro, he said that the filament of the lamp had no dimension exceeding $3 \mathrm{~mm}$. Redman said that it was depressing that most of the scintillation measured by Hall occurred at great heights. How could this be reconciled with the known correlation between seeing and conditions near the earth's surface, wind and ordinary weather? Would these results be of meteorological significance? Rösch said that a clear distinction should be kept between intensity variations such as those measured by Hall which undoubtedly arose at great heights, and those effects leading to a deterioration of definition of stellar images, which could arise much nearer the telescope.

Hall then showed a series of films of the movement of shadow patterns and the movement of stellar images. In discussion Ellison asked if any calibrations had been done on these films so that a quantitative comparison could be made of intensity and positional variations. The answer was in the negative. With regard to the films of shadow patterns, it was stated that 64 frames/sec. would not quite stop the motion of the markings. Keller showed a picture of Hosfeld's which showed that I/roo sec. stopped the motion and indicated that the elongations seen in photographs of longer exposure, were just trailed images.

The next statement was by Zwicky and was read, in his absence, by Bidelman. He said that work was being carried out on a series of experiments for the investigation of scintillation phenomena.

He showed slides showing trailed, focused star images having a time resolution as small as $\mathrm{rO}^{-7} \mathrm{sec}$. They were obtained by propelling a film across the prime focus of the 200 -inch reflector with speeds up to $30 \mathrm{~m}$. $/ \mathrm{sec}$. They showed knots, splits and lateral excursions. 
Similarly trailed extra focal images showed mottlings because parts of the beams were focused in different extra focal positions. With the extra focal image of Sirius I mm. in diameter, a time resolution of $0.5 \times 10^{-6} \mathrm{sec}$. was obtained with the 200-inch telescope and a plate movement of $30 \mathrm{~m}$./ $\mathrm{sec}$.

Trailed focused spectra were obtained having a time resolution less than $\mathrm{ro}^{-4} \mathrm{sec}$. They showed parallel intensity bands affecting all colours. Visual observations from aircraft showed that seeing improved fairly abruptly at a height of $7000 \mathrm{~m}$., at least as far as fluctuation of greater duration than $\mathrm{I} / 20 \mathrm{sec}$. were concerned.

He said that they had discovered aerial 'blobs' which acted as weak positive or negative lenses and which were of great durability. Their analysis was continuing. The effect of 'blobs', eddies, etc. decreased rapidly with angular distance, but in some cases were quite marked at angular separations of several minutes of arc. Compression waves caused by jet planes, lightning and shells or bullets could be followed practically over the whole sky.

In discussion, A. G. Wilson said that until it was known whether Zwicky's blobs of great duration were located in non-adiabatic turbulent regions of the atmosphere, it might be erroneous to associate them with any type of scintillation phenomena.

A. G. Wilson next spoke of work at Lowell on planetary photography. He showed examples of star trails on nights of different seeing and pointed out that under good seeing conditions a portion of an image could move I sec. of arc in I/6o sec. of time. Work on binary stars had shown that the bulk of scintillation occurred above $40,000 \mathrm{ft}$. It was important to note that, under certain conditions, there was $100 \%$ correlation between the excursions of the components of a binary with 6 minutes of arc separation.

Next, Bidelman spoke of work at the Lick Observatory to see whether there was any correlation between the estimates of seeing made with the 36 -inch refractor and the presence of a Jet Stream overhead. The fact that there was a very good correlation between the presence of the Stream and the absence of any observing record at all (due to clouds, fog, or high wind) makes it difficult to get any data on this question. However, it could be said that during the last few years the seeing was never very good when the Stream was near Mt Hamilton, and from the data available it appeared that there may be a fairly good relation between bad seeing and the presence of the Stream. Whether this was a direct relation is difficult to ascertain. The occasional observation of considerably different seeing in different parts of the sky at approximately the same time does point to a rather direct effect of the Jet Stream on seeing. Bidelman suggested that a comparative study of seeing at several observatories located a few hundred miles apart would aid in the solution of this question.

In discussion, Wilson stated that Carpenter, at the Steward Observatory, Tucson, was also investigating the relation between seeing and the presence of the Jet Stream, and that he had found somewhat similar results.

Finally, Butler showed slides of the distribution of intensity within star images. They had been obtained by scanning the images at the Cassegrain focus (focal length $54 \mathrm{ft}$.) of the Edinburgh 36 -inch reflector under various conditions of seeing.

It appeared from preliminary observations that under conditions of moderate seeing about $20 \%$ of the light in the image entered a slit of width $50 \mu$. He also showed as a function of time, a curve of the intensity of light actually entering the slit of a spectrograph under operational conditions. The fluctuations due to image movements and due to guiding errors were clearly visible and showed that optimum efficiency, even with a $90 \mu$ slit, required automatic or semi-automatic guiding. In reply to a question by Hynek, Butler suggested that as there was some possibility of confusion over nomenclatureparticularly in the definition of 'seeing' and 'scintillation'-the matter be left to the sub-commission. It would be one of the first things they would have to consider.

The meeting of the Commission then concluded. 